\title{
Case Report \\ Unusual Localization of an Emergent Bacterium, Raoultella ornithinolytica
}

\author{
Michele Cavaliere, ${ }^{1}$ Guido Bartoletti, ${ }^{1}$ Pasquale Capriglione, ${ }^{1}$ \\ Antonella Miriam Di Lullo $\mathbb{D}^{1},{ }^{1}$ Gaetano Motta, ${ }^{2}$ Maurizio Iengo, ${ }^{1}$ and Elena Cantone $\mathbb{D}^{1}$ \\ ${ }^{1}$ Department of Neuroscience, Reproductive and Odontostomatological Sciences-ENT Section, University "Federico II", \\ Naples, Italy \\ ${ }^{2}$ Otorhinolaryngology, Head and Neck Surgery Unit, Department of Mental and Physical Health and Preventive Medicine, \\ Università degli Studi della Campania Luigi Vanvitelli, Naples, Italy
}

Correspondence should be addressed to Elena Cantone; elenacantone@libero.it

Received 20 October 2019; Revised 4 February 2020; Accepted 25 February 2020; Published 30 March 2020

Academic Editor: Georgios D. Kotzalidis

Copyright (c) 2020 Michele Cavaliere et al. This is an open access article distributed under the Creative Commons Attribution License, which permits unrestricted use, distribution, and reproduction in any medium, provided the original work is properly cited.

\begin{abstract}
Raoultella ornithinolytica is a bacterium belonging to the family Enterobacteriacae. It is a rare but emergent cause of human pathologies especially in immunocompromised patients. We described the first case in the literature of isolated external otitis sustained by Raoultella ornithinolytica in an immunocompetent host. A 54-year-old Caucasian man with a history of previous myringoplasty came to our attention reporting otalgia and otorrhea. We performed right ear swab for culture examination, meanwhile we started empirical therapy with topic administration of neomycin, without any clinical improvement. The cultural examination showed the presence of a Raoultella ornithinolytica infection. After ten days of treatment with oral ciprofloxacin and topic levofloxacin, there was the complete resolution of pain and inflammation. Raoultella ornithinolytica must be taken into consideration as an emergent cause of human infection, also in case of external otitis. Infection can be severe and can occur both in immunocompromised and in immunocompetent hosts. Culture test is mandatory to choose the proper therapy and avoid potential severe complications.
\end{abstract}

\section{Introduction}

Raoultella ornithinolytica is a gram-negative, capsulate, aerobic, and nonmotile bacterium belonging to the family Enterobacteriacae. Three species of Raoultella, initially classified as Klebsiella, exist, R. electrica, R. planticola, and R. terrigena $[1,2]$. These bacteria have been isolated from aquatic environments, fish, and ticks, where it can produce histamine because of histidine decarboxylase enzyme and can contaminate food, especially not well conserved fish and pork [3]. Histamine toxicity produces symptoms that include flushing of skin, headache, pruritus, and abdominal cramping [3]. This rare but emergent bacterium can cause a wide spectrum of clinical manifestations. For instance, human infections caused by $R$. ornithinolytica are rare but can be responsible for bacteraemia, especially in patient with malignancies and immunodeficiencies [4, 5]. Furthermore, $R$. ornithinolytica is responsible for urinary, bile tract, tracheal, bronchial, and lungs infections in patients with immunodeficiency. The majority of cases reported in the literature are community acquired infections, especially nosocomial [6]. Neonatal sepsis is rare; however, it can be severe if not treated [7], as well as septic arthritis of the temporomandibular joint. In a case report after an early but temporary response to the antibiotic therapy, this bacterium leads to a complete demolition of the articulation [8]. $R$. ornithinolytica not only expresses $\beta$-lactamase, which provides resistance to commonly used $\beta$-lactam antibiotics but can also acquire genes for multi-drug resistance $[8,9]$.

Only a few ear, nose, and throat cases complaining difficulty in swallowing, pain in the throat, rhinosinusitis, and dysphonia are described in the literature. We described 
the first case in the literature of isolated external otitis (EO) sustained by $R$. ornithinolytica in an immunocompetent host.

\section{Case Presentation}

A 54-year-old Caucasian man presented to our hospital with a 7-day history of right otalgia and purulent otorrhea without any systemic symptoms or fever. In order to control pain, the patient was previously treated with acetaminophen alone for 3 days.

There was no significant family, social, or medical history except for a myringoplasty for tympanic membrane perforation of the right ear performed 7 years earlier and a septoplasty performed 5 years earlier.

On physical examination, the patient's blood pressure was found to be $125 / 70 \mathrm{mmHg}$, pulse was 80 beats per minute, temperature was $36^{\circ} \mathrm{C}$, and respiratory rate was 16 breaths per minute.

We observed pain on tragus pressure, whereas no paralysis of cranial nerves was observed. The right outer ear canal contained purulence with significant hyperemia and edema of skin. Tympanic membrane was impossible to visualize due to the narrowing of the outer ear canal. The rest of the head and neck examination, including the left ear, was normal.

Laboratory data at admission revealed a hemoglobin level of $14 \mathrm{~g} / \mathrm{dl}$ and white blood cell count of 14.700 per microliter with $70 \%$ neutrophils. Inflammation markers were high: erythrocyte sedimentation rate (ESR) was $25 \mathrm{~mm} /$ $\mathrm{h}$ (normal range $0-22 \mathrm{~mm} / \mathrm{h}$ ) in the first hour, and the level of C-reactive protein (CRP) was $15 \mathrm{mg} / \mathrm{L}$ (normal range $<3.0 \mathrm{mg} / \mathrm{L})$.

The computed tomography scan did not show soft alterations nor mastoid, skull base, and bony changes suggestive of malignant external otitis (Figure 1). We performed right ear swab for culture examination; meanwhile, we started empirical therapy with topic administration of neomycin for 7 days, without any clinical improvement.

The cultural examination showed the presence of a $R$. ornithinolytica infection. $R$. ornithinolytica isolates were identified with MALDI-TOF MS [10].

On the seventh day, as soon as the susceptibility profile of R. ornithinolytica was available (Table 1), we started a systemic therapy with ciprofloxacin $500 \mathrm{mg}$ twice a day for 10 days, and topic therapy using 5 ear drops composed by $3 \%$ boric acid in $70 \%$ alcohol and 5 drops of levofloxacin twice a day for 10 days, after that we observed a complete resolution of symptoms. In addition, the right ear otomicroscopy showed a dry cavity with only a small granulation on the upper anterior quadrant of the tympanic membrane that disappeared after ten more days of boric acid drops. The six months follow-up did not show recurrence.

\section{Discussion}

We describe a case of culture-confirmed $R$. ornithinolytica external otitis, defined as diffuse inflammation of the external ear canal, which may also involve the pinna or

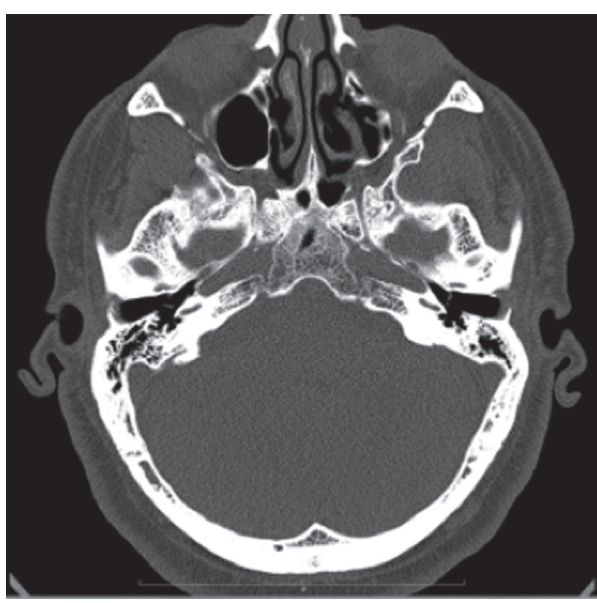

FIgUre 1: The computed tomography (CT) did not show bone alterations or changes suggestive of malignant external otitis.

TABle 1: Susceptibility of $R$. ornithinolytica.

\begin{tabular}{lcc}
\hline \multicolumn{3}{c}{ Susceptibility of $R$. ornithinolytica } \\
Antimicrobial & MIC & Interpretation \\
\hline Ampicillin & $\geq 32$ & $R$ \\
Amoxicillin/clavulanic acid & $\geq 32$ & $R$ \\
Piperacillin/tazobactam & $>8$ & $R$ \\
Gentamicin & $\leq 1$ & $S$ \\
Amikacin & $>16$ & $R$ \\
Nalidixic acid & $>8$ & $R$ \\
Ciprofloxacin & $\leq 0.25$ & $S$ \\
Fosfomycin & 64 & $R$ \\
Cefoxitin & $\geq 64$ & $R$ \\
Cefixime & $>16$ & $R$ \\
Ceftazidime & $>16$ & $R$ \\
Ceftriaxone & $>32$ & $R$ \\
Trimethoprim/sulfamethoxazole & $>32$ & $R$ \\
\hline
\end{tabular}

MIC: minimum inhibitory concentration.

tympanic membrane, in an immunocompetent man. $R$. ornithinolytica is a rare gram-negative aerobic bacillus belonging to the Enterobacteriaceae family. It represents an emergent cause of human infections. Virulence factors involved in the pathogenicity of $R$. ornithinolytica are its ability to adhere to human tissues converting histidine to histamine and to form biofilms [11-14]. Generally, $R$. ornithinolytica infections are observed in patients with diabetes, immunodepression, or oncological diseases [15]. These infections can cause sepsis, arthritis, urinary tract, and throat impairments; they can be severe and rarely occur in an immunocompetent host [11]. There are only a few cases of EO caused by $R$. ornithinolytica reported in the literature, but no one isolated [11]. To our knowledge, this is the first case described of isolated external otitis sustained by $R$. ornithinolytica in an immunocompetent host. All in all, $R$. ornithinolytica is not considered a virulent pathogen per se, but its ability to develop antibiotic resistance can cause severe complications $[4,7,16]$. Hence, the need is to identify the type of bacterium in order to set up the most appropriate therapeutic protocol. This is precisely what we did in our case, especially in the light of the failure of the initially 
attempted empirical therapy with neomycin. The reported case, based on the susceptibility profile, presented multidrug resistance (Table 1).

Moreover, in our patient, we did not find any correlation with previous myringoplasty surgery.

The American Academy of Otolaryngology-Head and Neck Surgery Foundation (AAO-HNSF) developed a clinical practice guideline recommending the use of topical preparations for initial therapy of diffuse, uncomplicated EO, whereas systemic antimicrobial therapy should be used in case of extension outside of the ear canal or in the presence of risk factors like diabetes, prior radiotherapy, or immune compromise [17]. However, in our case report we started a systemic therapy based on antimicrobial susceptibility for the lack of therapeutic response to topical therapy.

In our opinion, the culture test is mandatory to choose the proper therapy and to avoid potential severe complications as sepsis, arthritis, and meningitis. Indeed, systemic therapy prevents spreading of the infection, whereas topic therapy medicates the local district. We also recommend toilette of outer ear canal before starting and during the treatment, to provide higher efficacy of the local treatment.

In conclusion, otologists should take into consideration the infection sustained by this bacterium because they can be severe and can occur not only in immunocompromised patients but also, although rarely, in an immunocompetent host.

In our opinion, physicians should be aware of the high rates of antimicrobial resistance of R. ornithinolytica as demonstrated by antimicrobial susceptibility in our case report. Further studies have to be done to understand the entity of the diffusion of this bacterium.

\section{Conflicts of Interest}

The authors declare that they have no conflicts of interest.

\section{References}

[1] M. Drancourt, C. Bollet, A. Carta, and P. Rousselier, "Phylogenetic analyses of Klebsiella species delineate Klebsiella and Raoultella gen. nov., with description of Raoultella ornithinolytica comb. nov., Raoultella terrigena comb. nov. and Raoultella planticola comb. nov," International Journal of Systematic and Evolutionary Microbiology, vol. 51, no. 3, pp. 925-932, 2001.

[2] V. P. Morais, M. T. Daporta, A. F. Bao, M. G. Campello, and G. Q. Andrés, "Enteric fever-like syndrome caused by Raoultella ornithinolytica (Klebsiella ornithinolytica)," Journal of Clinical Microbiology, vol. 47, no. 3, pp. 868-869, 2009.

[3] Y.-C. Lee, Y.-F. Chen, Y.-L. Huang, H.-F. Kung, T.-Y. Chen, and Y.-H. Tsai, "Hygienic quality, adulteration of pork and histamine production by Raoultella ornithinolytica in milkfish dumpling," Journal of Food and Drug Analysis, vol. 24, no. 4, pp. 762-770, 2016.

[4] P. Seng, B. M. Boushab, F. Romain et al., "Emerging role of Raoultella ornithinolytica in human infections: a series of cases and review of the literature," International Journal of Infectious Diseases, vol. 45, pp. 65-71, 2016.

[5] Y. Hadano, M. Tsukahara, K. Ito, J. Suzuki, I. Kawamura, and H. Kurai, "Raoultella ornithinolytica bacteremia in cancer patients: report of three cases," Internal Medicine, vol. 51, no. 22, pp. 3193-3195, 2012.

[6] M. Boattini, A. Almeida, C. Cardoso et al., "Infections on the rise: Raoultella spp., clinical and microbiological findings from a retrospective study, 2010-2014," Infectious Diseases, vol. 48, no. 1, pp. 87-91, 2016.

[7] A. Abbas and I. Ahmad, "First report of neonatal early-onset sepsis caused by multi-drug-resistant Raoultella ornithinolytica," Infection, vol. 46, no. 2, pp. 275-277, 2018.

[8] J. Levorova, V. Machon, A. Guha, and R. Foltan, "Septic arthritis of the temporomandibular joint caused by rare bacteria Raoultella ornithinolytica," International Journal of Oral and Maxillofacial Surgery, vol. 46, no. 1, pp. 111-115, 2017.

[9] A. Sękowska, "Raoultella spp.—clinical significance, infections and susceptibility to antibiotics," Folia Microbiologica, vol. 62, no. 3, pp. 221-227, 2017.

[10] P. Seng, C. Abat, J. M. Rolain et al., "Identification of rare pathogenic bacteria in a clinical microbiology laboratory: impact of matrix-assisted laser desorption ionization-time of flight mass spectrometry," Journal of Clinical Microbiology, vol. 51, no. 7, pp. 2182-2194, 2013.

[11] M. Singh, I. Kaur, D. K. Mundi, and A. Kaur, "ENT infection caused by Raoultella ornithinolytica," Nigerian Journal of Clinical Practice, vol. 20, no. 7, pp. 914-917, 2017.

[12] E. Cantone, R. Negri, E. Roscetto et al., "In vivo biofilm formation, gram-negative infections and TAS2R38 polymorphisms in CRSw NP patients," The Laryngoscope, vol. 128, no. 10, pp. E339-E345, 2018.

[13] E. Cantone and M. Iengo, "Effect of sodium hyaluronate added to topical corticosteroids in chronic rhinosinusitis with nasal polyposis," American Journal of Rhinology \& Allergy, vol. 30, no. 5, pp. 340-343, 2016.

[14] M. Cavaliere, A. M. Di Lullo, E. Cantone et al., "Cholesteatoma vs. granulation tissue: a differential diagnosis by DWI-MRI apparent diffusion coefficient," European Archives of Oto-Rhino-Laryngology, vol. 275, no. 9, pp. 2237-2243, 2018.

[15] S. Chun, J. W. Yun, H. J. Huh, and N. Y. Lee, "Clinical characteristics of Raoultella ornithinolytica bacteremia," Infection, vol. 43, no. 1, pp. 59-64, 2015.

[16] A. Kayode, P. Okunrounmu, A. Olagbende, O. Adedokun, A. W. Hassan, and G. Atilola, "High prevalence of multiple drug resistant enteric bacteria: evidence from a teaching hospital in Southwest Nigeria," Journal of Infection and Public Health, no. 19, pp. 30294-30301, 2019, In press.

[17] R. M. Rosenfeld, S. R. Schwartz, C. R. Cannon et al., "Clinical practice guideline," Otolaryngology-Head and Neck Surgery, vol. 150, no. 1_suppl, pp. S1-S24, 2014. 